hep-th/0306139

AEI 2003-044

UUITP-09/03

ITEP-TH-31/03

\title{
Stringing Spins and Spinning Strings
}

\author{
N. Beisert ${ }^{a}$, J.A. Minahan $^{b}$, M. Staudacher ${ }^{a}$ And K. Zarembo ${ }^{b, *}$ \\ ${ }^{a}$ Max-Planck-Institut für Gravitationsphysik \\ Albert-Einstein-Institut \\ Am Mühlenberg 1, D-14476 Golm, Germany \\ nbeisert, matthias@aei.mpg.de \\ ${ }^{b}$ Department of Theoretical Physics \\ Uppsala University \\ Box 803, SE-751 08, Uppsala, Sweden \\ joseph.minahan, konstantin.zarembo@teorfys.uu.se
}

\begin{abstract}
We apply recently developed integrable spin chain and dilatation operator techniques in order to compute the planar one-loop anomalous dimensions for certain operators containing a large number of scalar fields in $\mathcal{N}=4$ Super Yang-Mills. The first set of operators, belonging to the $\mathrm{SO}(6)$ representations $[J, L-2 J, J]$, interpolate smoothly between the BMN case of two impurities $(J=2)$ and the extreme case where the number of impurities equals half the total number of fields $(J=L / 2)$. The result for this particular $[J, 0, J]$ operator is smaller than the anomalous dimension derived by Frolov and Tseytlin hep-th/0304255 for a semiclassical string configuration which is the dual of a gauge invariant operator in the same representation. We then identify a second set of operators which also belong to $[J, L-2 J, J]$ representations, but which do not have a BMN limit. In this case the anomalous dimension of the $[J, 0, J]$ operator does match the Frolov-Tseytlin prediction. We also show that the fluctuation spectra for this $[J, 0, J]$ operator is consistent with the string prediction.
\end{abstract}

*also at ITEP, Moscow, Russia. 


\section{Introduction}

In the $A d S_{5} \times S^{5}$ conjecture [1,2,3], it was argued that the anomalous dimension of a generic gauge invariant operator scales as

$$
\gamma \sim(\lambda)^{1 / 4} \sqrt{\ell}
$$

where $\lambda=g_{\mathrm{YM}}^{2} N$ is the 't Hooft coupling and $\ell$ is the string level [2]. However, there are some operators which do not behave like (1.1) even for large values of $\lambda$ 4. One example are the half-BPS operators whose dimensions are protected by supersymmetry.

Another example are the BMN operators, which are nearby to the half-BPS operators [5]. These operators have been heavily studied [6, 7, 8, 9, 10, 11, 12, 13, 14, 15, 16, 17, 18, 19, 20, 21, 22. For these operators, one starts with a half-BPS operator $\operatorname{Tr}\left(Z^{J}\right)$ where $Z=\phi_{5}+i \phi_{6}$, and then inserts "impurities". The impurities, e.g. the scalars $X=\phi_{1}+i \phi_{2}$ and $Y=\phi_{3}+i \phi_{4}$, are inserted in various positions in the operator

$$
\mathcal{O}=\operatorname{Tr} Z \ldots Z X Z \ldots Z X Z \ldots Z Y Z \ldots Z
$$

Alternatively, one can think of this as an operator with $L$ scalar fields, where $L-J$ of the original Z's are converted to one of the other scalars. One-loop effects mix the operators among themselves and the operators with definite scaling dimension, along with their anomalous dimensions, are obtained by diagonalizing a matrix. This matrix of anomalous dimensions is particularly easy to compute by analyzing the action of the dilatation operator [19, 21, even in the non-planar sector and at higher-loops.

In the BMN limit, where the number of impurities is small compared to $L$, it was shown that the eigenstates of this dilatation operator are isomorphic to particles on a circle of length $L$, with the number of particles equal to the number of impurities. With a small number of impurities one can safely ignore the particle interactions and use the dilute gas approximation. The total momentum of the particles has to be zero in order to guarantee cyclicity of the trace, and one finds that the one-loop anomalous dimension behaves like

$$
\gamma=\frac{\lambda}{8 \pi^{2}} \sum_{i} \epsilon\left(p_{i}\right)=\frac{\lambda}{8 \pi^{2}} \sum_{i}\left(p_{i}\right)^{2}
$$

where $p_{i}$ are the individual particle momenta and $\epsilon\left(p_{i}\right)$ can be thought of as their energies. Quantization on a circle of length $L$ then gives

$$
\gamma=\frac{\lambda}{2 L^{2}} \sum_{i}\left(n_{i}\right)^{2}
$$

where the $n_{i}$ are integers.

Remarkably, these results can be directly related to a string calculation on $A d S_{5} \times S^{5}$ [23, 24, 25, 26, 27, 28, 29, 30,31,32,33,34, 35, 36, since one can choose $\lambda$ large but $L$ also large such that $\lambda^{\prime}=\lambda / L^{2}$ is small. One then finds a one to one map of the BMN operators to string states propagating in the plane wave limit [37, 38] of $A d S_{5} \times S^{5}$. These states can be written as

$$
\prod_{i}\left(\alpha_{n_{i}}^{\mu_{i}}\right)^{\dagger}\left|p_{+}\right\rangle
$$


where $p_{+}=L$ in appropriate units. In the limit of small $\lambda^{\prime}$, the results then match with (1.4).

More recently, it has been suggested that other operators can be compared with semiclassical string calculations [39, 40,41,42,43, 44]. The operators of [42, 44] have order $L$ impurities, with each impurity carrying momentum of order $1 / L^{2}$. The anomalous dimension is now of order $\lambda / L$. The corresponding string states are expected to have order $L$ creation operators.

In particular, Frolov and Tseytlin considered operators that have $J^{\prime}$ of the $Z$ scalars and an equal number $J$ of the $X$ and $Y$ scalars [44. Assuming that these are highest weight states, one finds that these are operators in the $\left[0, J^{\prime}-J, 2 J\right]$ representation of $\mathrm{SO}(6)$ if $J^{\prime} \geq J$, or the $\left[J-J^{\prime}, 0, J+J^{\prime}\right]$ representation if $J>J^{\prime}$, up to conjugation. In the extreme case where $J^{\prime}=0$, one finds a semiclassical result which is twice the BMN prediction [44. That there is a difference from BMN is not surprising - the density of impurities is of order 1 , so the dilute gas approximation is naively expected to break down.

The case of a very large operator with a high density of impurities is not easily accessible by field theoretic means. Nevertheless we can still make progress in computing the one-loop planar anomalous dimension for these types of operators. This is because the planar one-loop dilatation operator for scalars, representing the interaction found in [12, is isomorphic to the Hamiltonian for an integrable system [18] and one can then use all of the machinery that goes along with it.

In particular, if one restricts the operators to be composed only of $Z$ and $X$ scalars, then the problem simplifies dramatically ${ }^{1}$. This is because the dilatation operator can be mapped directly to the Hamiltonian of the Heisenberg spin chain, which was solved long ago by Bethe. The $Z$ scalars inside $\mathcal{O}$ are the up spins and the $X$ scalars are the down spins. The field theory representation $[J, L-2 J, J]$ corresponds to a configuration of total spin $L / 2-J$. The eigenstates for the spin chain can be found by solving the Bethe equations. These are easy to solve for the case of two spin flips ${ }^{2}$, but for more than two flips these are in general only solvable numerically.

Solving for a finite number of $L / 2$ spin flips with total spin 0 looks almost hopeless. However, in the large $L$ limit, there is some hope of making progress since here one can convert the Bethe equations to an integral equation. This is how the ground state of the antiferromagnetic Heisenberg spin chain was solved; it has total spin 0 and energy proportional to $-L$.

To make contact with the proposal of 44 the low-lying states of the ferromagnetic spin chain with total spin 0 and energy proportional to $1 / L$ are of particular interest. Here, the antiferromagnetic ground state is the state with the highest energy. In contrast, the semiclassical string configuration is expected to correspond to the state of the lowest energy, or at least much lower energy.

In this paper we solve this problem for $[J, L-2 J, J]$ representations where $J$ and $L$ are assumed to be large. This corresponds to operators of the form (modulo ordering of

\footnotetext{
${ }^{1}$ Operators with only $Z$ and $X$ scalars are special as they only mix amongst themselves due to charge conservation 21 .

${ }^{2}$ In the context of $\mathcal{N}=4 \mathrm{SYM}$ these are the finite $J$ versions of the two-impurity BMN operators which were identified and diagonalized in [16].
} 
fields)

$$
\mathcal{O}=\operatorname{Tr} Z^{L-J} X^{J}+\ldots
$$

As in the solution for the antiferromagnet ground state, this is solved by converting the Bethe equations to an integral equation. However, unlike the case of the antiferromagnetic ground state, the Bethe roots do not lie on the real line, but instead extend into the complex plane.

We consider two classes of $[J, L-2 J, J]$ operators. The first class has two contours of Bethe roots symmetric about the imaginary axis which we call the double contour solution. The second class has all Bethe roots on the imaginary axis which we call the imaginary root solution. These latter Bethe states are "singular" in the sense that the Bethe equations need to be regularized. One of the main results of this paper is to identify the $[J, 0, J]$ (spin 0 ) imaginary root solution as the SYM dual of the string solution found by Frolov and Tseytlin 44].

The agreement between the SYM and semiclassical string theory calculations is remarkable: the anomalous dimension exactly coincides with the energy of the string, and the spectrum of nearby operators coincides with the spectrum of small fluctuations around the classical string solution. Furthermore, the semiclassical analysis indicates that there is an instability in the string solution. This is consistent with our analysis, where we show that the imaginary root solution is not the lowest energy $[J, 0, J]$ state. Instead we show that the double contour solution is the ground state for this representation ${ }^{3}$.

We first consider the double contour Bethe state. In the limit of small $J$, this state approaches the BMN states with $J / 2$ each of $\alpha_{-1}$ and $\alpha_{+1}$ oscillators. We are able to solve this problem by first considering the unphysical region where $J<0$, and then analytically continuing to $J>0$. The problem then reduces to solving for two elliptic equations which is easily done numerically to any desired precision. For the case where $J=L / 2$, and in the strict $J \rightarrow \infty$ limit, we find that the anomalous dimension is given by

$$
\gamma=\frac{\lambda J}{L^{2}} \beta=\frac{\lambda}{4 J} \beta, \quad \text { with } \beta=0.7120321458 \ldots
$$

The result in (1.7) is bigger than the BMN result (1.4) where one finds $\beta=1 / 2$. However, (1.7) is smaller than the semiclassical string result $\beta=1$ in [44. Hence, we conclude that this double contour solution is not the gauge dual to the semiclassical string.

To help us find the gauge dual to the semiclassical string, we compare our result in (1.7) to actual numerical computations of $[J, 0, J]$ ground states. We find very close agreement when $J$ is even. However, when $J$ is odd, we find that while the result in (1.7) appears correct, the configuration of Bethe roots is completely different from the even case. In this case there are two curving contours in the complex plane connected by a straight line of roots along the imaginary axis. It would be interesting to solve the integral equation describing this distribution of Bethe roots.

\footnotetext{
${ }^{3}$ Strictly speaking, the double contour solution is valid only for even $J$ while the imaginary root solution is valid only for odd $J$. Indeed, the ground state for odd $J$ has its Bethe roots in a completely different configuration than the double contour, and we have yet to determine the even $J$ analog of the imaginary root solution. However, in the thermodynamic limit (large $L$ ), there should be no distinction between even and odd and we will provide strong evidence for this in Sec. 3 .
} 
Nevertheless, it turns out that there are also solutions where all Bethe roots lie on the imaginary axis. With all imaginary roots, the relative coefficients in the sum over states for the spin chain (or gauge invariant operator) are real. For $J$ small compared to $L$, the roots form two overlapping "strings" where the separation between roots is very close to $i / 2$. If $J$ is of the same order as $L$, then the outer roots spread out. If we then take the limit of large $J$ and $L$, we can reduce the Bethe equations to an integral equation which is very similar to the equation found by Douglas and Kazakov for two-dimensional QCD on the sphere [45,46]. We find that when $J=L / 2$ there is a critical point and for this value the anomalous dimension matches the prediction in 44. Unlike the case of the double contour, as the number of roots is reduced, the state does not approach a BMN state.

To further verify that this imaginary root solution is the gauge dual of the semiclassical string, we consider spinless fluctuations about the solution. This is done by pulling roots off of the imaginary axis and onto (or close to) the real axis. Here we find a spectrum that is consistent with that found in 44].

In section two we solve for the solution with a double contour of roots in the large $L$ limit. We find $\gamma$ as a function of $L$ and show that it gives (1.7). In section three we compare this result to actual numerical computations of $[J, 0, J]$ states. Here we show the surprising difference between the even and odd configurations. In section four we consider and solve for the imaginary root solution in the large $L$ limit. We show that it matches the predictions in [4] for the anomalous dimension and the fluctuation spectrum. In section five we consider an analogous solution to an $\mathrm{SO}(6)$ singlet configuration and show that it matches a semiclassical prediction made in [42]. In section six we present our conclusions.

\section{The double contour solution}

In this section we describe the ground state for the $[J, L-2 J, J]$ representations. Strictly speaking, this solution is valid only if $J$ is even. If $J$ is odd and $L>2 J$, then the true ground state is doubly degenerate. But it approaches the double contour solution in the limit of large $L$. In the next section, we will argue that if $J$ is odd and $L=2 J$, then the ground state will have a completely different root configuration than the solution described in this section. However, the anomalous dimension appears to be the same.

We begin with the Bethe equations, which $\mathrm{read}^{4}$

$$
\left(\frac{u_{j}+i / 2}{u_{j}-i / 2}\right)^{L}=\prod_{\substack{k=1 \\ k \neq j}}^{J} \frac{u_{j}-u_{k}+i}{u_{j}-u_{k}-i}
$$

Here $L$ is the total length of the spin chain and $J$ is the number of impurities ${ }^{5}$. The complex numbers $u_{j}$ are the rapidities of the upside down spins $\left(2 u_{j}=\cot p_{j}\right.$, where $p_{j}$ is the momentum of $j$ th impurity). Normally the rapidities are real, but the impurities

\footnotetext{
${ }^{4}$ For a nice review see 47 .

${ }^{5}$ Note that this connotation has recently been introduced in the BMN literature. This is different from impurities in condensed matter theory, where one would rather speak, equivalently, of "excitations", "particles" or "magnons". Or even simpler, "spins down"!
} 
can form bound states in which case their rapidities acquire imaginary parts. In the literature on the Bethe ansatz the bound states of impurities are usually referred to as "strings". We shall study bound states of a macroscopically large number of impurities and shall argue that these large "strings" in the Heisenberg model are dual to strings in $A d S_{5} \times S^{5}$ spinning around the five-sphere. The state with $J$ impurities belongs to the representation $[J, 2 L-J, J]$ of $\mathrm{SO}(6)$ and we define the filling fraction

$$
\alpha=\frac{J}{L} \leq \frac{1}{2}
$$

We can also write down the logarithmic version of the Bethe equations

$$
L \log \frac{u_{j}+i / 2}{u_{j}-i / 2}=2 \pi i n_{j}+\sum_{\substack{k=1 \\ k \neq j}}^{J} \log \frac{u_{j}-u_{k}+i}{u_{j}-u_{k}-i}
$$

where the numbers $n_{j}$ reflect the ambiguity of the branches of the logarithm. These numbers can be interpreted as the lattice momentum of the $j$-th 'particle'.

Since the spin chain corresponds to an operator with a trace, we are only interested in states that are invariant under shifts. This forces us to set the trace condition

$$
\prod_{j=1}^{J} \frac{u_{j}+i / 2}{u_{j}-i / 2}=1
$$

The eigenvalues of the planar dilatation operator can be found by solving the system of Bethe equations for the roots $u_{j}(j=1, \ldots, J)$ [18. This is somewhat cumbersome for chains of small length, but very advantageous for long chains where a direct diagonalization [19,21] is no longer feasible. The planar anomalous dimensions are then given to one-loop, for general $L$ and $J$, by

$$
\Delta=L+\gamma \quad \text { with } \quad \gamma=\frac{\lambda}{8 \pi^{2}} \sum_{j=1}^{J} \frac{1}{u_{j}^{2}+1 / 4}
$$

In the "thermodynamic limit" where $L \rightarrow \infty$, the roots are of order $u_{j}=\mathcal{O}(L)$. Let us therefore rescale them as

$$
u_{j} \rightarrow L u_{j}
$$

Then one easily proves that the Bethe equations (2.3) simplify for $L \rightarrow \infty$ to

$$
\frac{1}{u_{j}}=2 \pi n_{j}+\frac{2}{L} \sum_{\substack{k=1 \\ k \neq j}}^{J} \frac{1}{u_{j}-u_{k}} .
$$

Here, the term on the left corresponds to a global potential, while the second term on the right corresponds to pairwise repulsion of roots. As is standard when taking the thermodynamic limit of the Bethe equations, eq. (2.7) can be conveniently turned into an integral equation.

In order to turn the Bethe equations (2.7) into a single integral equation we need to understand the structure of the distribution of Bethe roots in the complex plane. 


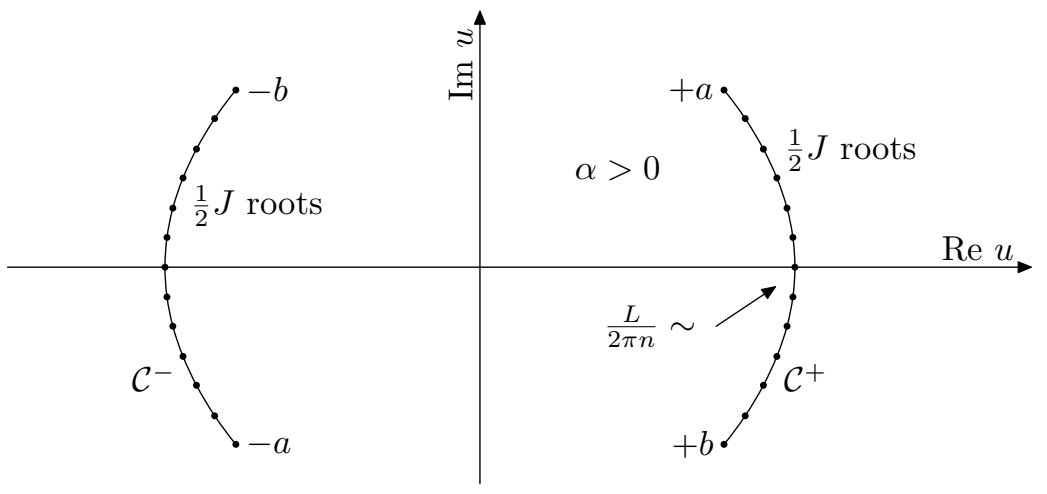

Figure 1: Bethe roots. For large $L$ the roots condense into two cuts

To gain some intuition, let us concentrate on the case that $L$ and $J$ are large but the filling fraction $\alpha \ll 1 / 2$. We will also assume for now that $J$ is even. The trickier case of odd $J$ will be discussed (but not analyzed to the end) below. As can be seen from (2.7), to a reasonable approximation the Bethe roots may be placed around the positions $u_{i}=1 /\left(2 \pi n_{i}\right)$, as long as no two $n_{i}$ are the same. However, we wish to lower the energy as much as possible, which means setting all $n_{i}$ to \pm 1 with an equal number of each to satisfy the condition in (2.4). The interaction term in (2.7) pushes the roots apart. It is well known that the roots are pushed in the imaginary direction and form "strings" roughly parallel to the imaginary axis with the separation between the $u_{i}$ of order $1 / \sqrt{L}\left[18\right.$. It is clear from the Bethe equations that given a root $u_{i}$, there has to be a root $u_{j}=u_{i}^{*}$, therefore the distribution of roots is invariant under reflection about the real axis.

It was also shown that there was a $1 / L$ correction in the real direction towards the imaginary axis, where the correction is larger the further the root is from the real line. Finally, it was argued that placing the roots like this led to corrections to $\gamma$ which were of order $1 / L^{3}$, while changing the branch gives a $1 / L^{2}$ correction. Hence, at least for small $J / L$, it is energetically favorable to evenly distribute the roots on the \pm 1 branches and hence the distribution looks like that in Fig. 1.

If $J$ is of order $L$, then adding a root to the heavily populated \pm 1 branches costs an energy of order $1 / L^{2}$. However, we will assume that for $\alpha \sim 1 / 2$ it is still favorable to place the roots in these branches. We can also make a slight generalization and place all of the roots in the branches $\pm n$. From the semiclassical string perspective, we expect this to correspond to a string that winds $n$ times around itself. The net effect of this is to multiply $\sqrt{\lambda}$, the effective string tension, by a factor of $n$.

Assuming that $J$ is still even, the roots are distributed so that they are symmetric under reflection about the imaginary axis. Hence we only need to consider the first $J / 2$ roots $u_{j}$ with $j=1, \ldots, J / 2$, since we can now assign $u_{j+J / 2}=-u_{j}$. Based on our previous argument, we now assume that $n_{j}=n=1,2, \ldots$. Let us introduce a density

$$
\rho(u)=\frac{2}{J} \sum_{k=1}^{J / 2} \delta\left(u-u_{k}\right)
$$

describing the distribution of roots with positive real part in the complex plane. This is expected to turn into a smooth function in the limit of large $L$, with a continuous 

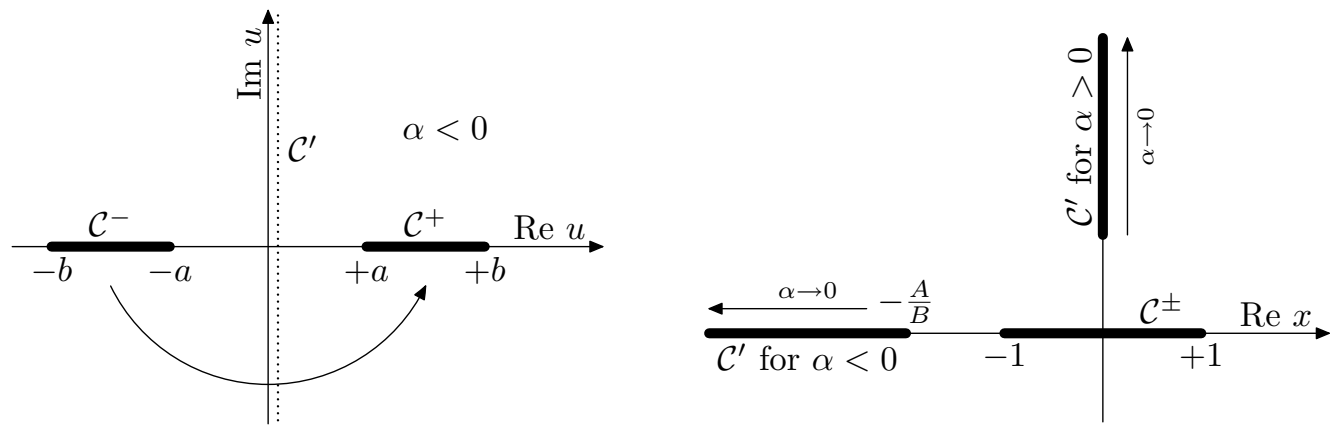

Figure 2: Cuts flip for $\alpha<0$. Both cuts can be mapped to one $\mathcal{C}^{ \pm}$via the map $u^{2}=A+B x$.

support tracing a curve $\mathcal{C}^{+}$(see Fig. 1). The density is normalized to one:

$$
\int_{\mathcal{C}^{+}} d u \rho(u)=1
$$

One easily verifies that the Bethe equations (2.7) can be reexpressed with the help of the density as

$$
f_{\mathcal{C}^{+}} d v \rho(v) \frac{v^{2}}{u^{2}-v^{2}}=\left(\frac{1}{2 \alpha}-1\right)-\frac{\pi n}{\alpha} u
$$

Here the integral has to be understood in the principal part sense, as we need to omit the case $k=j$ in equation (2.7). The anomalous dimension, $c f$ eq. (2.5) becomes (remember the rescaling $\left.u_{j} \rightarrow L u_{j}\right)$

$$
\gamma=\frac{\lambda}{8 \pi^{2}} \frac{\alpha}{L} \int_{\mathcal{C}^{+}} d u \frac{\rho(u)}{u^{2}}
$$

Therefore all we need to do is invert the singular integral equation (2.10); this yields the density, and therefore, in light of eq. (2.11), the anomalous dimension. Luckily, similar equations have appeared previously in the case of large $N$ matrix models (which, incidentally, are also integrable models of sorts). In particular, equation (2.10) is a close relative of the equations describing the solution of Kostov's $\mathrm{O}(n)$ multi-matrix model ${ }^{6}$. An inconvenient feature of (2.10) is that the precise shape of the contour $\mathcal{C}^{+}$is unknown. The situation can be improved by first solving the equation in the unphysical regime $\alpha<0$, corresponding to a negative number of down spins. Here we expect the cuts to lie on the real axis, as is shown in the matrix model problems. The contour $\mathcal{C}^{+}$becomes the real interval $[a, b]$ while the mirror contour $\mathcal{C}^{-}$turns into the flipped interval $[-b,-a]$, as in the left half of Fig. 2. This way we merely need to determine the endpoints $a, b$ but not the shape of the contour. After solving the equation for general negative $\alpha$ we can subsequently analytically continue back to the physical regime $\alpha \in[0,1 / 2]$. Next, let us apply the same folding map as in the case of the $\mathrm{O}( \pm 2)$ matrix model [4]:

$$
u=\sqrt{A+B x} \quad \text { with } \quad A=\frac{1}{2}\left(b^{2}+a^{2}\right) \quad \text { and } \quad B=\frac{1}{2}\left(b^{2}-a^{2}\right)
$$

This maps the two cuts $[a, b]$ and $[-b,-a]$ on top of each other, and to the interval $[-1,1]$. Our equation becomes,

$$
f_{-1}^{1} d y \rho(y) \frac{\sqrt{A+B y}}{x-y}=\left(\frac{1}{\alpha}-2\right)-\frac{2 \pi n}{\alpha} \sqrt{A+B x}
$$

\footnotetext{
${ }^{6}$ see 48 and references therein.
} 
where $\rho(u) \equiv \rho(x(u))$. Equation (2.13) is a standard one-matrix model (or airfoil) equation with a non-polynomial potential involving $\sqrt{A+B x}$. The new cut $\mathcal{C}^{\prime}$ with branchpoints at $x=\infty$ and $x=-A / B$ appears due to the above folding map, as shown in the right part of Fig. 2. The latter branchpoint lies for $\alpha<0$ to the left of -1 on the negative real axis, while for $\alpha>0$ it sits on the imaginary axis. The solution for the density is immediately obtained by an inverse finite Hilbert transform:

$$
\rho(x)=-\frac{1}{\pi^{2}} \frac{\sqrt{1-x^{2}}}{\sqrt{A+B x}} f_{-1}^{1} d y \frac{1}{x-y} \frac{1}{\sqrt{1-y^{2}}}\left[\left(\frac{1}{\alpha}-2\right)-\frac{2 \pi n}{\alpha} \sqrt{A+B y}\right] .
$$

It simplifies to

$$
\rho(x)=\frac{2 n}{\pi \alpha} \frac{\sqrt{1-x^{2}}}{\sqrt{A+B x}} f_{-1}^{1} d y \frac{1}{x-y} \frac{\sqrt{A+B y}}{\sqrt{1-y^{2}}} .
$$

This is nearly the solution to our problem, except that we still need to determine the parameters $A, B$ as a function of the filling fraction $\alpha$. These are determined from the normalization condition (2.9) which reads in the new variables

$$
\frac{B}{2} \int_{-1}^{1} d x \frac{\rho(x)}{\sqrt{A+B x}}=1
$$

and the further condition

$$
\int_{-1}^{1} d x \frac{1}{\sqrt{1-x^{2}}}\left[\left(\frac{1}{\alpha}-2\right)-\frac{2 \pi n}{\alpha} \sqrt{A+B x}\right]=0
$$

which ensures the positivity of the density on the cut. It simplifies to

$$
\int_{-1}^{1} d x \frac{\sqrt{A+B x}}{\sqrt{1-x^{2}}}=\frac{1}{n}\left(\frac{1}{2}-\alpha\right) .
$$

This completes the solution: $A$ and $B$ are determined through the two equations (2.16), (2.18), which one could express through elliptic integrals of the first and second kind, while the density is given by equation (2.15), which could be found explicitly in terms of an elliptic integral of the third kind. However, as we are chiefly interested in the anomalous dimension (2.11), we can simply eliminate the density. Plugging (2.15) into both the normalization condition (2.16) and the expression for the anomalous dimensions (2.11), we respectively find, after exchanging orders of integration,

$$
\int_{-1}^{1} d x \frac{1}{\sqrt{1-x^{2}} \sqrt{A+B x}}=\frac{1}{2 n} \frac{1}{\sqrt{A^{2}-B^{2}}}
$$

and

$$
\gamma=\frac{\lambda}{16 \pi^{2} L}\left(\frac{1-2 \alpha}{\sqrt{A^{2}-B^{2}}}-\frac{A}{A^{2}-B^{2}}\right) .
$$

Thus $\gamma$ is explicitly given by the parameters $A, B$ which in turn are implicit functions of $\alpha$ and $n$, as stated in eqs. (2.18), (2.19). It is now a straightforward exercise to invert these equations in terms of a series in $\alpha$ to any desired order, one finds

$$
\gamma=\frac{\lambda n^{2} J}{2 L^{2}}\left(1+\frac{1}{2} \alpha+\frac{3}{8} \alpha^{2}+\frac{21}{64} \alpha^{3}+\frac{159}{512} \alpha^{4}+\frac{315}{1024} \alpha^{5}+\frac{321}{1024} \alpha^{6} \ldots\right)
$$


While we derived this result for negative $\alpha$, we see that the function is perfectly analytic at $\alpha=0$ and we can immediately analytically continue to the correct Bethe phase $\alpha \in$ $[0,1 / 2]$. The leading order $\mathcal{O}(\alpha)$ result correctly reproduces the dilute gas approximation (1.4) and the famous BMN result $\lambda n^{2} / L^{2}$ for $J=2$. Let us also note that the leading $1 / L$ correction to the BMN limit

$$
\gamma=\frac{\lambda n^{2} J}{2 L^{2}}\left(1+\frac{J+2}{2 L}+\mathcal{O}\left(1 / L^{2}\right)\right)
$$

was obtained in 18; it agrees with the $\mathcal{O}\left(\alpha^{2}\right)$ term in (2.21) and thus yields a nice check of our method. The above series (2.21) converges rather rapidly in the interval $\alpha \in[0,1 / 2]$ allowing us to obtain the anomalous dimension to arbitrary precision for any $\alpha$. We can also expand the anomalous dimension around $\alpha=1 / 2$, where we find

$$
\gamma=\frac{\lambda}{2 L}[0.7120-1.0916(1-2 \alpha)+\ldots]
$$

A plot of these results in presented in Fig. 3 in the next section.

An alternative method for solving (2.18) and (2.19) is to analytically continue $\alpha$ first, thus moving $B$ onto the imaginary axis. For small values of $\alpha, A$ is positive, but decreases as $\alpha$ is increased and eventually changes sign for a critical value of $\alpha$. At this value the branch point on the imaginary axis touches the cut along the real line.

To avoid this collision, we simply deform the integration on the real line to an integration along the cut on the imaginary axis. If we define $C=-i B$, then the equation in (2.19) becomes

$$
2 \int_{A / C}^{\infty} d y \frac{1}{\sqrt{1+y^{2}} \sqrt{C y-A}}=\frac{1}{2 n} \frac{1}{\sqrt{A^{2}+C^{2}}}
$$

Defining a new variable $\omega=\sqrt{C y-A}$, (2.24) can be reexpressed as

$$
\int_{-\infty}^{\infty} d \omega \frac{1}{\sqrt{\left(\omega^{2}+A\right)^{2}+C^{2}}}=\frac{1}{2 n} \frac{1}{\sqrt{A^{2}+C^{2}}} .
$$

We then do the same with (2.18), although now there will be a divergent piece that needs to be subtracted off. Deforming the contour, redefining variables and subtracting off the infinite piece, we find

$$
\int_{-\infty}^{\infty} d \omega\left(1-\frac{\omega^{2}}{\sqrt{\left(\omega^{2}+A\right)^{2}+C^{2}}}\right)=\frac{1}{n}\left(\frac{1}{2}-\alpha\right) .
$$

If we set $A$ to zero, then the elliptic integrals reduce to ordinary integrals and we can find an analytic solution. In this case

$$
\begin{aligned}
& \alpha=\frac{1}{2}-\frac{4 \pi^{2}}{(\Gamma(1 / 4))^{4}} \approx 0.2715<\frac{1}{2} \\
& \gamma=\frac{\lambda n^{2}}{2 \pi L}
\end{aligned}
$$


Hence $A$ becomes negative before $\alpha$ reaches $\alpha=1 / 2$. Assuming that $A<0$ and letting $\eta=-C / A$ we define the integrals

$$
\begin{aligned}
& I_{1}(\eta)=\int_{-\infty}^{\infty} d z\left(1-\frac{z^{2}}{\sqrt{\left(z^{2}-1\right)^{2}+\eta^{2}}}\right) \\
& I_{2}(\eta)=\int_{-\infty}^{\infty} \frac{d z}{\sqrt{\left(z^{2}-1\right)^{2}+\eta^{2}}} .
\end{aligned}
$$

We then find from (2.25) and (2.26) that

$$
\alpha=\frac{1}{2}\left(1-\frac{1}{\sqrt{1+\eta^{2}}} \frac{I_{1}(\eta)}{I_{2}(\eta)}\right) .
$$

While it is possible to solve this for all physical values of $\alpha$, let us only consider the case $\alpha=1 / 2$, which corresponds to setting $I_{1}$ to zero. This is easily done numerically, where one finds $\eta=1.16220056$. From this it is straightforward to show that the value for $\gamma$ is that in (1.7) and that the values for $A$ and $C$ are

$$
A=-0.086987288 \quad C=0.10109667 .
$$

In terms of our original variable $u$, we see then that the branch points are at

$$
u= \pm 0.1523 \pm 0.3319 i \text {. }
$$

The exact distribution of the roots is found by starting from the branch points and finding the contour where $d u \rho(u)$ is positive definite. The positivity of the density unambiguously determines the shape of the contour [49]. Here, $\rho(u)$ is the analytic continuation of (2.15). In terms of $x$, this means

$$
\frac{B \rho(x)}{\sqrt{A+B x}} d x
$$

is positive definite. While we have not solved for this over the whole contour, we have determined that the slope of the contour away from the upper right branch point in (2.31) is -0.743 . Hence the distribution has the general form in Fig. 11.

\section{Comparison of results}

We now compare our results of the previous section to the exact anomalous dimensions of operators of the form (1.6) for reasonably large values of $L$. These can be obtained directly using the dilatation operator or using the exact Bethe equations.

\subsection{Dilatation operator}

For given $L$ and $J$ we collect all operators of the form $\operatorname{Tr} Z^{L-J} X^{J}+\ldots$ and act on them with the dilatation operator [21]. In this way the matrix of anomalous dimensions is generated and we subsequently diagonalize it. A slight complication is that the set of 


\begin{tabular}{|cc|cc|}
\hline$J$ & $\gamma\left[\lambda J / L^{2}\right]$ & $J$ & $\gamma\left[\lambda J / L^{2}\right]$ \\
\hline 2 & 0.6079271019 & 3 & 0.9118906528 \\
4 & 0.6576949106 & 5 & 0.8416441952 \\
6 & 0.6759273535 & 7 & 0.8155060662 \\
8 & 0.6848144787 & 9 & 0.7973649389 \\
\hline
\end{tabular}

Table 1: Numerical values for the lowest eigenvalues in $[J, 0, J]$

operators (1.61) comprises not only the operators in the representation $[J, L-2 J, J]$, but also the ones for smaller values of $J$. These additional eigenvalues have to be removed as some of them are generically smaller than the one we are interested in. An easy way to achieve this is to repeat the analysis for the operators $\operatorname{Tr} Z^{L-J+1} X^{J-1}+\ldots$ and remove the corresponding eigenvalues ${ }^{7}$. What remains are exactly the anomalous dimensions of the operators in the representation $[J, L-2 J, J]^{8}$.

In this way we have obtained the anomalous dimensions for all operators of length

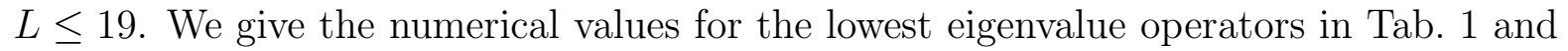
Fig. 3. We note that the behavior of the anomalous dimensions is significantly different for even and odd values of $J$. We will therefore treat them separately in what follows.

Already for $J=8$ the accuracy is very good compared to the numerically exact result (1.7) at $J \rightarrow \infty$. We can improve the estimate for $J \rightarrow \infty$ by linearly expanding around $J=\infty$,

$$
\gamma=(a+b / J+\ldots) \frac{\lambda J}{L^{2}}
$$

We fit the coefficients $a, b$ to the available data for $J=4,6,8$ and get

$$
\gamma=(0.712-0.22 / J+\ldots) \frac{\lambda J}{L^{2}}
$$

The estimates depend on the selected set of data and the fit function, overall the agreement with (1.7) seems to be within $1 \%$.

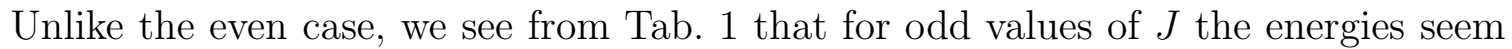
to approach an asymptotic value of $\mathcal{O}\left(\gamma L^{2} / J\right)$ from above. A fit for odd $J$ with (3.1) yields $\gamma=(0.743+0.49 / J) \lambda J / L^{2}$. While the even and odd case seems to asymptote to a different value, this could be due to the paucity of data points in the fit. In the thermodynamic limit where $J$ is large, it is hard to believe there should be a distinction between even and odd. Further down in this section, we will present evidence that indeed the difference vanishes, see (3.9).

We can make further observations on the obtained data for $L \leq 19$. An important feature of the one-loop planar dilatation generator or of integrable spin chains in general is that states may come in pairs, see [21. We observe that

- the energy of the ground state is $\mathcal{O}(1 / L)$,

- for even $J$ the ground state is unpaired,

\footnotetext{
${ }^{7}$ One of the benefits of the Bethe ansatz is that states of a lower representation are easily identified, they have roots at infinity, and discarded.

${ }^{8}$ For the representation $[9,1,9]$ of $\mathrm{SU}(4)$ this involved 4862 states. Out of these, 884 belong to the representation $[9,1,9]$, the other belong to $[J, 19-2 J, J]$ with $J<9$.
} 


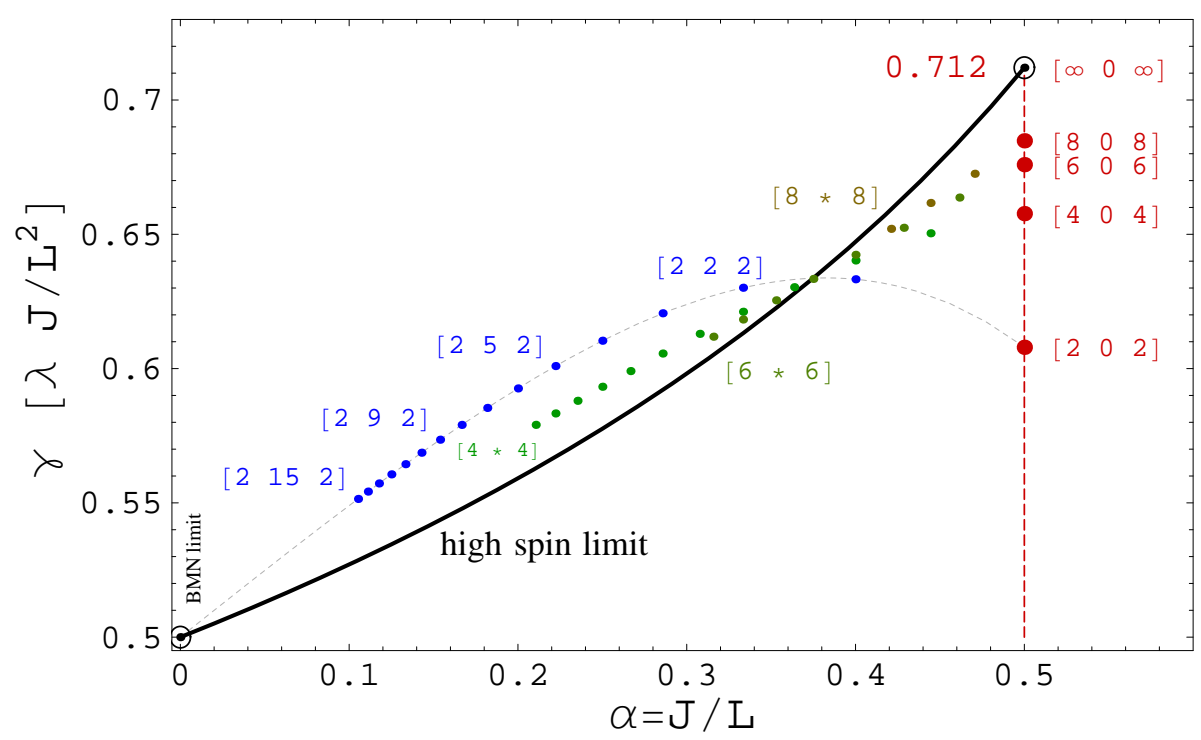

Figure 3: States of lowest energy in $[J, L-2 J, J], J$ even. The plot shows how the energy per spin flip increases from the dilute gas, $\alpha=0$, to the maximum filling $\alpha=1 / 2$. Discrete values for $L \leq 19$ are obtained as eigenvalues of the dilatation generator and the curve for $L \rightarrow \infty$ represents the solution to the Bethe equations. The states with $J=2$ were found in [16].

- for odd $J$ the ground state is paired unless $J=L / 2$,

- for odd $J$ the energy of the lowest unpaired state is $\mathcal{O}(1 / J)$,

- for odd $J$ and $L$ all states are paired.

It is also interesting to investigate the operators of lowest energy. For $\alpha=1 / 2$ the operators are

$$
\mathcal{O}=a \operatorname{Tr} Z^{J} X^{J}+\sum_{m, n=1}^{J-1} b_{m, n} \operatorname{Tr} Z^{m} X^{n} Z^{J-m} X^{J-n}+\ldots
$$

where $a$ and $b$ are mixing coefficients. We find that $a$ is by far the most dominant coefficient. The coefficients $b_{m, n}$ are less important, while the remaining coefficients are almost negligible. This is in analogy to the Weiss domains configuration of a ferromagnet. We find that alike spins cluster in domains with only the domain walls contributing to the energy. For a minimal energy solution the number of domains must therefore be as small as possible.

\subsection{Bethe roots}

The Bethe equations are non-linear equations of multiple variables $u_{j}$. It is therefore a difficult task to find solutions to them. A numerical solution requires the knowledge of the approximate position of the roots $u_{j}$. It would therefore be useful to know how the roots arrange in general. We have worked out solutions for small values of $J$ and $L$ to obtain some intuition in this respect.

First of all we note that the Bethe equations (2.1), the trace condition (2.4) and the energy (2.5) are invariant under negation of roots $u_{j} \rightarrow-u_{j}$. Therefore each solution 
$\left\{u_{j}\right\}$ has a partner $\left\{-u_{j}\right\}$ of equal energy unless $\left\{u_{j}\right\}=\left\{-u_{j}\right\}$. This is the same pairing discussed in the previous subsection. Here we will concentrate on the unpaired states. This is also simplifies the analysis because now there are only half as many equations to solve.

For unpaired solutions we must distinguish between an even and an odd number of roots $J$. For even $J$ all roots come in pairs $u_{J / 2+j}=-u_{j}$ and the trace condition (2.4) is automatically satisfied. The important feature for odd $J$ is that one of the roots must be zero, while the others come in pairs. At first glance, this would seem to violate the trace condition since the zero Bethe root contributes a factor -1 while $u_{j}$ and $-u_{j}$ together contribute a factor of 1 .

There is one way around this problem, which is to also place two Bethe roots at the singular positions $\pm i / 2$. These roots need to be regularized since the Bethe equations diverge for these values ${ }^{9}$. Hence we may assume that there are three roots

$$
u_{J}=0, \quad u_{J-1, J-2}=i( \pm 1 / 2+\varepsilon \pm \delta),
$$

where $\delta=\mathcal{O}\left(\varepsilon^{L}\right)$. The Bethe equations associated to the singular roots and the trace condition are satisfied in the limit $\varepsilon \rightarrow 0$. The Bethe equation associated to the zero root implies that $L$ must be even. This is in agreement with the observation of the last subsection. The contribution of the special roots to $\gamma$ is finite and is given by

$$
\gamma=\frac{\lambda}{8 \pi^{2}} \lim _{\varepsilon \rightarrow 0}\left(4-\frac{1}{\varepsilon+\varepsilon^{2}}-\frac{1}{-\varepsilon+\varepsilon^{2}}\right)=\frac{3 \lambda}{4 \pi^{2}}
$$

If $J=3$ then there are no other roots and this state corresponds to the special three impurity operators discussed in [21].

We have determined the root configurations for all unpaired states of the $[J, 0, J]$ spin chain for $2 \leq J \leq 7$. The positions of the roots were found by rewriting the Bethe equations in polynomial form. These equations were combined into a single polynomial equation of one variable using the resultant. All Bethe roots are then among the solutions to this equation. We summarize our findings in Tab. 2 and Tab. 5, 6] at the end of this paper.

We make the following observations. All the roots of a given mode number $n$ form a vertical string of roots approximately at the real coordinate $L / 2 \pi n$. This is in agreement with [18. What is different is the distance of roots in the imaginary direction. For a low density of roots $\alpha$, the roots of equal mode number $n$ should be separated by $i \mathcal{O}(\sqrt{L})$. For a high density the separation is very close to $i$ if the roots are close to the real axis, but spreads out as we move away from the axis.

\subsection{The odd ground state}

Since the three roots in (3.4) contribute a finite amount to $\gamma$, the other roots must somehow cancel off most of this contribution to leave a $\gamma$ of order $\mathcal{O}(1 / L)$. We note that if a Bethe root is on the imaginary axis with $\left|u_{i}\right|>1 / 2$, then this root contributes a negative amount to $\gamma$.

\footnotetext{
${ }^{9}$ The existence of these singular solutions to the Bethe equations was recognized by Bethe himself 50 and has drawn certain attention with regard to the completeness of the Bethe ansatz $51,52,53,54$.
} 


\begin{tabular}{|c|c|lll|c|}
\hline$J$ & $\gamma\left[\lambda J / L^{2}\right]$ & \multicolumn{3}{|c|}{ Bethe roots } & $n$ \\
\hline 2 & 0.607927 & 0.288675 & & 1 \\
\hline 3 & 0.911891 & 0 & $\pm i / 2$ & $2 *$ \\
\hline 4 & 0.657695 & $( \pm 0.463265 \pm 0.502294 i)$ & 11 \\
& 1.104851 & $\pm 1.025705 i$ & \pm 0.041309 & 03 \\
& 2.290302 & \pm 0.525012 & \pm 0.129473 & & 13 \\
\hline 5 & 0.841644 & 0 & $\pm i / 2$ & $\pm 0.998506 i$ & $3 * 1$ \\
& 1.291310 & 0 & $\pm i / 2$ & $\pm 1.570673 i$ & $4 * 0$ \\
& 2.289413 & 0 & $\pm i / 2$ & \pm 0.638965 & $4 * 1$ \\
& 3.176721 & 0 & $\pm i / 2$ & \pm 0.236124 & $4 * 2$ \\
\hline
\end{tabular}

Table 2: States of $[J, 0, J]$

Let us examine more closely the Bethe roots for relatively small odd $J$. Assuming the presence of the singular roots in (3.4), the [5,0,5] state has two more roots which are the negative of each other and which have to be either real or imaginary in order to be invariant under the shift operator. Hence, the Bethe equations in (2.1) reduce to a single equation

$$
\left(\frac{u+i / 2}{u-i / 2}\right)^{8}=\frac{(u+i)(u+3 i / 2)}{(u-i)(u-3 i / 2)} .
$$

One then finds that the solution that gives the entry in Tab. 1 is imaginary and very close to $i$. A similar analysis for the ground state of the $[7,0,7]$ operator shows that two more roots are placed very close to $\pm 3 i / 2$ and that the other two roots move even closer to $\pm i$. This pattern continues up to and including the $[11,0,11]$ operator, where roots are added near half integer imaginary values and where the lower roots move closer to the half integers as higher roots are added.

The half-integer periodicity of imaginary roots can be explained by the same argument that leads to the string hypothesis for the structure of Bethe states in the thermodynamic limit [47. If $u_{n}=i q_{n}$, then the Bethe equations in (2.1) take the form

$$
\left(\frac{q_{n}+1 / 2}{q_{n}-1 / 2}\right)^{L}=\prod_{m \neq n} \frac{q_{n}-q_{m}+1}{q_{n}-q_{m}-1} .
$$

For $q_{n}$ of order one and positive, the exponentially large factor on the 1.h.s. of (3.7) has to be compensated by a small denominator on the r.h.s., which arises when two roots are separated by an amount which is close to 1 . Since we are starting with the roots in (3.4), this tends to put the new roots close to the half-integers. We can also see that increasing $L$ forces the lower roots closer to the half-integers.

One can also see that with this configuration of roots $\gamma$ will be of order $\mathcal{O}(1 / L)$. If we assume that the roots are equally distributed along half-integer imaginary values, then 


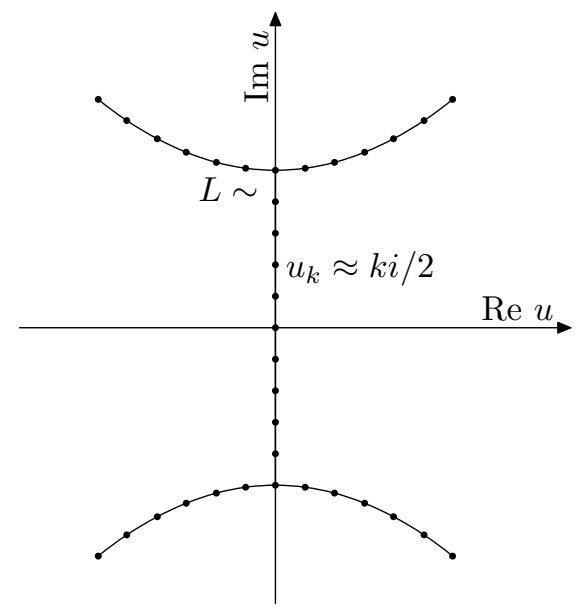

Figure 4: Distribution of roots for the odd ground state.

\begin{tabular}{|rc|rc|rr|}
\hline$J$ & $\gamma\left[\lambda J / L^{2}\right]$ & $J$ & $\gamma\left[\lambda J / L^{2}\right]$ & $J$ & $\gamma\left[\lambda J / L^{2}\right]$ \\
\hline 3 & 0.911891 & 13 & 0.773992 & 23 & 0.748305 \\
5 & 0.841644 & 15 & 0.766364 & & \\
7 & 0.815506 & 17 & 0.760374 & & \\
9 & 0.797365 & 19 & 0.755558 & & \\
11 & 0.783974 & 21 & 0.751605 & & \\
\hline
\end{tabular}

Table 3: Odd ground state energies for $[J, 0, J]$

from (2.5) we find

$$
\begin{aligned}
\gamma & =\frac{\lambda}{8 \pi^{2}}\left(6-8 \sum_{j=2}^{\frac{J-1}{2}} \frac{1}{n^{2}-1}\right) \\
& =\frac{\lambda}{8 \pi^{2}}\left(6-8 \sum_{j=2}^{\infty} \frac{1}{n^{2}-1}+8 \sum_{j=\frac{J+1}{2}}^{\infty} \frac{1}{n^{2}-1}\right) \\
& \approx \frac{8}{\pi^{2}} \frac{\lambda}{4 J} .
\end{aligned}
$$

The prefactor in (3.8) is quite close to the values in Tab. 1. It is also in agreement with the observation of Sec. 3.1 that the energy of odd unpaired solutions is of order $\mathcal{O}(1 / J)$.

However, for large enough $J$ and $q_{n}$ we expect this picture to break down since the l.h.s. of (3.7) will then be of order 1 . In fact, for the $[13,0,13]$ case we found that the ground state has 9 roots on the imaginary axis and 4 split off from it into the complex plane. For higher values of $J$, more roots will break off from the imaginary axis, see Fig. 4. In Tab. 3 we show the ground state values for $\gamma$ up to $J=23$. If we fit the higher data points starting with $J=13$ to the curve in (3.1) we find

$$
\gamma=(0.715+0.77 / J) \frac{\lambda J}{L^{2}}
$$

indicating that the odd result is asymptoting to (1.7). Hence the even and odd cases 


\begin{tabular}{|c|ccc|}
\hline$J$ & $\gamma_{1, J}\left[\lambda J / L^{2}\right]$ & $\gamma_{2, J}\left[\lambda J / L^{2}\right]$ & $\gamma_{3, J}\left[\lambda J / L^{2}\right]$ \\
\hline 3 & 0.911891 & & \\
5 & 0.841644 & 1.291309 & \\
7 & 0.815506 & 1.033470 & 1.684344 \\
9 & 0.797365 & 0.963623 & 1.210401 \\
11 & 0.783974 & 0.931003 & 1.068883 \\
13 & & 0.907919 & 1.008200 \\
15 & & 0.888306 & 0.976885 \\
\hline
\end{tabular}

Table 4: $[J, 0, J]$ states with roots on the imaginary axis

are approaching the same result even though the configuration of Bethe roots is totally different.

\subsection{States with imaginary roots}

Even though they will not give the smallest value for $\gamma$, there still can be solutions to the Bethe equations with all roots on the imaginary axis. These remain interesting objects, because they still correspond to some string states in $A d S_{5} \times S^{5}$. What one finds is that the lower roots are very close to the half-integers, but the higher roots tend to spread out. In fact, for a given value of $J>3$, there seem to be multiple solutions of imaginary Bethe roots. Results for $\gamma$ where the state has all imaginary roots are shown in Tab. 4 .

Let us consider all imaginary solutions for a given value of $J$, we label them $U_{k, J}$. Comparing this to the case $J+2$, it seems that for each solution $U_{k, J}$ there is a corresponding solution $U_{k, J+2}$. In the examples considered in Tab. 4 this solution has a similar distribution of roots with two additional roots. Often, these roots are also on the imaginary axis, in some cases, however, they can spread out into the complex plane. For example, this happens in $U_{1,13}$, see the discussion in the last subsection. Although the roots cease to be exclusively on the imaginary axis, the energy $\gamma_{1,13}$ continues to follow the extrapolated trajectory, see Tab. 3. However, when we discard these states we notice that the value of $\gamma L^{2} / J$ jumps up, see Tab. 4.

We can therefore assume the following picture: Trajectories of imaginary solutions $U_{k, J}$ appear at some value of $J$. At a higher value of $J$ some roots of $U_{k, J}$ split off of the imaginary axis. When we discard such states, which have a relatively low energy, the state with the minimum energy will be $U_{k+1, J}$ and the lowest $\gamma L^{2} / J$ lurches upward. But one can also see in Tab. 4 that as $J$ becomes large, there is some indication that the result is approaching the semiclassical result of Frolov and Tseytlin for the spinning string. We show that this is the case in the following section.

\section{The gauge dual of the semiclassical string}

In this section, we show that the state with all imaginary roots has the expected properties of the dual to the semiclassical string in 44. We first show that the anomalous dimension has the expected behavior in the limit of large $L$. We then consider spinless fluctuations about the solution and show that they too have the expected behavior. 


\subsection{A solution for imaginary roots in the large $L$ limit}

In the previous section, we saw evidence that if all roots have the form $u_{j}=i q_{j} L$ with $q_{j}$ real, then the roots close to the real line are very close to the imaginary half integers, but those further away begin to spread out. We will call those roots separated by half integers the "condensate". When $L$ becomes large (2.3) becomes

$$
\frac{1}{q_{j}}=\frac{2}{L} \sum_{\substack{k=-\frac{J-1}{2} \\ k \neq j}}^{\frac{J-1}{2}} \frac{1}{q_{j}-q_{k}}
$$

where (4.1) is valid for $q_{j}$ outside the condensed region. Note in particular that there is no integer on the r.h.s. due to a branch ambiguity. The equation in (4.1) can be rewritten as an integral equation

$$
\frac{1}{q}=f d q^{\prime} \frac{\sigma\left(q^{\prime}\right)}{q-q^{\prime}}
$$

which is valid for $q$ outside the condensate and where the root density $\sigma\left(q^{\prime}\right)$ is given by

$$
\sigma\left(q^{\prime}\right)=\frac{2}{L} \sum_{k=-\frac{J-1}{2}}^{\frac{J-1}{2}} \delta\left(q^{\prime}-q_{k}\right)
$$

We now make the ansatz that the roots are condensed in the interior region, but start spreading out at some point of order $L$ from the origin. This means that the root density is of the form

$$
\sigma(q)= \begin{cases}4 & -s<q<s, \\ \widetilde{\sigma}(q) & s<q<t, \\ \widetilde{\sigma}(-q) & -t<q<-s, \\ 0 & q<-t \text { or } t<q\end{cases}
$$

where $t$ and $s$ are to be determined. Notice that this configuration is very similar to one considered by Douglas and Kazakov for the strongly coupled phase of $\mathrm{QCD}_{2}$ on the sphere [45, where the density also has a condensed region. The problem considered here is somewhat different since the $\mathrm{QCD}_{2}$ equations would have $q$ on the l.h.s. of (4.2) instead of $1 / q$. In terms of $\widetilde{\sigma}(q)$, (4.2) becomes

$$
\frac{1}{q}-4 \ln \frac{q+s}{q-s}=f_{s}^{t} d q^{\prime} \tilde{\sigma}\left(q^{\prime}\right)\left(\frac{1}{q-q^{\prime}}+\frac{1}{q+q^{\prime}}\right) .
$$

The solution of this integral equation can be written in the form

$$
\widetilde{\sigma}(q)=\frac{1}{\pi} \sqrt{\left(q^{2}-s^{2}\right)\left(t^{2}-q^{2}\right)}\left(-\frac{1}{q s t}+4 \int_{-s}^{s} \frac{d v}{(q-v) \sqrt{\left(s^{2}-v^{2}\right)\left(t^{2}-v^{2}\right)}}\right) .
$$

If we plug (4.6) back into (4.5), we see that $s$ and $t$ must satisfy a consistency condition

$$
4 \int_{-s}^{s} \frac{d v}{\sqrt{\left(s^{2}-v^{2}\right)\left(t^{2}-v^{2}\right)}}=\frac{1}{s t} .
$$


We also have the normalization condition for $\sigma(q)$

$$
\int d q \sigma(q)=8 s+2 \int_{s}^{t} d q \widetilde{\sigma}(q)=2 \alpha,
$$

where $\alpha$ is the previously defined filling factor. If we now insert (4.6) into (4.8) and use (4.7) we find the equation

$$
4 \int_{-s}^{s} \frac{d v v^{2}}{\sqrt{\left(s^{2}-v^{2}\right)\left(t^{2}-v^{2}\right)}}=1-2 \alpha .
$$

The anomalous dimension is found by using (2.5), (3.8) and (4.4), which gives

$$
\begin{aligned}
\gamma & =\frac{\lambda}{8 \pi^{2}}\left(6-2 \sum_{n=2}^{2 s L} \frac{4}{n^{2}-1}-2 \sum_{n=s L}^{\infty} \frac{1}{q_{n}^{2}}\right)+\mathcal{O}\left(1 / L^{2}\right) \\
& =\frac{\lambda}{8 \pi^{2}}\left(\frac{4}{s L}-\frac{1}{L} \int_{s}^{t} d v \frac{\widetilde{\sigma}(v)}{v^{2}}\right)+\mathcal{O}\left(1 / L^{2}\right)
\end{aligned}
$$

The integral inside (4.10) is found by using (4.6), (4.7) and (4.9), which leads to

$$
\gamma=\frac{\lambda}{32 \pi^{2} L}\left(\frac{1}{s^{2}}+\frac{1}{t^{2}}-2 \frac{1-2 \alpha}{s t}\right) .
$$

Let us now focus on the limiting case $\alpha=1 / 2$ which corresponds to the $[J, 0, J]$ representation. Using (4.9) and (4.7), we see that in the limit $\alpha \rightarrow 1 / 2, t$ and $s$ approach

$$
t \rightarrow \infty \quad s \rightarrow \frac{1}{4 \pi} .
$$

Therefore, setting $\alpha=1 / 2$, we find

$$
\gamma=\frac{\lambda}{2 L}=\frac{\lambda}{4 J}
$$

which is precisely the string theory prediction in [44]. Starting from this result we can also expand the energy around $\alpha=1 / 2$. We get

$$
\gamma=\frac{\lambda}{2 L}\left(1+8\left(\frac{1}{2}-\alpha\right)^{2}+24\left(\frac{1}{2}-\alpha\right)^{4}+96\left(\frac{1}{2}-\alpha\right)^{6}+408\left(\frac{1}{2}-\alpha\right)^{8}+\ldots\right)
$$

Interestingly, the energy is symmetric around $\alpha=1 / 2$ and reaches a maximum at $\alpha=$ $1 / 2$.

We can also see that the solutions for the $[J, L-2 J, J]$ representations do not approach a BMN limit when $J \ll L$. The easiest way to see this is to realize that the roots are all close to imaginary half-integers and so we can borrow the result in (3.8), giving ${ }^{10}$ $\gamma=2 \lambda / \pi^{2} J$ if $J \ll L$. Hence, for finite $J$ the anomalous dimension is finite in the large $L$ limit.

\footnotetext{
${ }^{10}$ The same results can be derived from eqs. (4.7), (4.8), (4.11) by noticing that $s \approx t \approx \alpha / 4$ and $t-s \sim \exp \left(-4 / \alpha^{2}\right)$ as $\alpha \rightarrow 0$.
} 
Note also that for $\alpha=1 / 2$, the root density simplifies to

$$
\widetilde{\sigma}(q)=4\left(1-\sqrt{1-\frac{1}{(4 \pi q)^{2}}}\right) .
$$

Hence, $4-\tilde{\sigma}(q)$ has a Wigner distribution in $1 / q$. This is like the critical point for Douglas-Kazakov, where the eigenvalue distribution changes to a Wigner distribution at the critical area.

\subsection{Fluctuations}

We can also show that the fluctuations of the solution in the previous section are consistent with the Frolov-Tseytlin prediction. The fluctuations we consider are spinless, that is, they do not change the $\mathrm{SO}(6)$ representation. Hence, these fluctuations are found by moving Bethe roots around in the complex plane, but not actually changing the net total of roots.

With this in mind, let us suppose that two roots are moved from the imaginary axis. If we assume that the remaining roots stay on the axis then in order to satisfy the trace condition, the two roots must be at $\pm \mu$ where $\mu$ is positive real. In the large $L$ limit, $\mu$ satisfies the equation

$$
\frac{1}{\mu}=2 \pi n+\int d q \frac{\sigma(q)}{\mu-i q},
$$

where we have again rescaled the roots by a factor of $L$. The integer $n$ corresponds to the different branch choice for the log. The root at $-\mu$ is on the $-n$ branch. We may also assume that the function $\sigma(q)$ is the same one computed in the previous section. Using $\sigma(q)=\sigma(-q)$ and the expression in (4.15), we obtain the equation

$$
\frac{1}{\mu}=2 \pi n+\int_{-\infty}^{\infty} d q \frac{4 \mu}{\mu^{2}+q^{2}}-4 \mu\left(\int_{-\infty}^{-s} d q+\int_{s}^{\infty} d q\right) \frac{\sqrt{q^{2}-s^{2}}}{q\left(q^{2}+\mu^{2}\right)}
$$

Deforming the contour, we then find

$$
\mu^{-1}=2 \pi \sqrt{n(n+4)} .
$$

Hence, we see that $n>0$ for $\mu$ to be positive real. The contribution of these roots to the anomalous dimension is

$$
\gamma_{\mu}=\frac{2 \lambda}{8 \pi^{2} L^{2} \mu^{2}}=\frac{n(n+4) \lambda}{L^{2}} .
$$

The roots at $\pm \mu$ also back react on the imaginary axis roots. To find this effect, we note that the roots at $\mu$ modify the equation in (4.5) to

$$
\frac{1}{q}-4 \ln \frac{q+s}{q-s}-\frac{4 q}{L\left(q^{2}+\mu^{2}\right)}=f_{s}^{t} d q^{\prime} \tilde{\sigma}\left(q^{\prime}\right)\left(\frac{1}{q-q^{\prime}}+\frac{1}{q+q^{\prime}}\right) .
$$

We can then solve for $\widetilde{\sigma}(q)$ with the new term on the 1.h.s. of the equation in (4.20). Thus, we have

$$
\int d q \sigma(q)=8 s+2 \int_{s}^{t} d q \widetilde{\sigma}(q)=2 \alpha-\frac{4}{L}
$$




$$
4 \int_{-s}^{s} \frac{d v}{\sqrt{\left(s^{2}-v^{2}\right)\left(t^{2}-v^{2}\right)}}=\frac{1}{s t}-\frac{4}{L} \frac{1}{\sqrt{\left(s^{2}+\mu^{2}\right)\left(t^{2}+\mu^{2}\right)}},
$$

and the same equation in (4.9). Hence, we see that the distribution still has $t \rightarrow \infty$ if $\alpha=1 / 2$, but the position of $\mathrm{s}$ is shifted to

$$
s \approx \frac{1}{4 \pi}\left(1-\frac{4}{L} \frac{1}{\sqrt{1+(4 \pi \mu)^{2}}}\right)
$$

We now compute the contribution of the imaginary roots to the anomalous dimension, where we find

$$
\gamma_{\mathrm{ir}}=\frac{\lambda}{32 \pi^{2} L}\left(\frac{1}{s^{2}}+\frac{1}{t^{2}}-2 \frac{1-2 \alpha}{s t}-\frac{2}{L \mu}\left(1-\frac{s t}{\sqrt{\left(s^{2}+\mu^{2}\right)\left(t^{2}+\mu^{2}\right)}}\right)\right) .
$$

If we now let $\alpha=1 / 2$, use (4.18) and (4.23), and then add (4.19) to (4.24), we find that the change in the anomalous dimension from (4.13) is

$$
\Delta \gamma=\frac{\lambda}{L^{2}}(n+2) \sqrt{n(n+4)} \text {. }
$$

This is the prediction of Frolov and Tseytlin, where our definition of $n$ is shifted by 2 from theirs, and with extra factor of 2 since this configuration is essentially two fluctuation quanta.

In their semiclassical analysis, Frolov and Tseytlin also identified an unstable mode, that is, a mode with an imaginary frequency. Since we are considering eigenstates of a Hermitian Hamiltonian we will not see this directly. It would seem that $\Delta \gamma$ is imaginary if $n=-1$ or -3 . However, in this case $\mu$ would also be imaginary. But the derivation of (4.18) assumed that $\operatorname{Re}(\mu)>0$. Hence the imaginary $\mu$ solution is not actually a solution to the Bethe equations.

On the other hand, we have already established that there are states with lower energy and in the large $L$ limit we would expect them to form a continuum, see Sec. 3.4 , Hence there can be a superposition of states centered about the imaginary root solution that will decay. If the superposition is spread out over solutions with width $\mathcal{O}\left(1 / L^{2}\right)$, then this will lead to frequencies with imaginary parts of the same order.

Even though the imaginary $\Delta \gamma$ do not correspond to solutions to the Bethe equations, they still give the predicted imaginary frequencies of Frolov and Tseytlin. Hence a possible interpretation is that the states constructed with these values of $\mu$ are precisely these superpositions of energy eigenstates.

Placing roots at $\pm \mu$ is consistent with level matching of the fluctuations. If we were to consider a finite number of roots removed from the axis and placed at $\pm \mu_{i}$, we may ignore their interactions among themselves and so the change in the anomalous dimension is additive. If we were to put two roots on the same cut $n$, they would split off from the real line [18, but the splitting is of order $1 / \sqrt{L}$ and so this can also be ignored at order $1 / L^{2}$. 
From the string perspective, there can be nonsymmetric configurations of roots that are still consistent with level matching. Assuming that such states exist, then the imaginary roots will also be nonsymmetric, which means that they must stray from the imaginary axis. It would be interesting to find these solutions. Also it would be interesting to find an analog of the semiclassical string configuration for even $J$.

\section{$5 \quad$ An $\mathrm{SO}(6)$ singlet solution}

It turns out that we can find a solution for an $\mathrm{SO}(6)$ singlet that is very similar to the solution in the last section. Once we consider the full $\mathrm{SO}(6)$, then it is necessary to introduce two other types of Bethe roots [18]. If we assume that all roots are imaginary and that the distribution of the two new sets of roots are the same, then the full $\mathrm{SO}(6)$ Bethe equations become

$$
\begin{aligned}
\left(\frac{q_{n}+1 / 2}{q_{n}-1 / 2}\right)^{L} & =\prod_{m \neq n} \frac{q_{n}-q_{m}+1}{q_{n}-q_{m}-1} \prod_{k}\left(\frac{q_{n}-r_{k}-1 / 2}{q_{n}-r_{k}+1 / 2}\right)^{2} \\
1 & =\prod_{k \neq j} \frac{r_{j}-r_{k}+1}{r_{j}-r_{k}-1} \prod_{m} \frac{r_{j}-q_{m}-1 / 2}{r_{j}-q_{m}+1 / 2}
\end{aligned}
$$

where $r_{j}$ refer to the new set of roots. Taking logs, rescaling by $L$ and converting these equations to integral equations, we find

$$
\begin{aligned}
& \frac{1}{q}=f d v \frac{\sigma(v)}{q-v}-f d v \frac{\omega(v)}{q-v} \\
& 0=f d v \frac{\omega(v)}{r-v}-\frac{1}{2} f d v \frac{\sigma(v)}{r-v}
\end{aligned}
$$

where $\sigma(q)$ is the same as (4.3) and $\omega(q)$ is

$$
\omega(r)=\frac{2}{L} \sum_{k} \delta\left(r-r_{k}\right) .
$$

Therefore, we have

$$
\frac{2}{q}=f d v \frac{\sigma(v)}{q-v}
$$

and so we have almost the same equation as in the previous section. The factor of 2 leads to the modified equations

$$
\begin{gathered}
4 \int_{-s}^{s} \frac{d v}{\sqrt{\left(s^{2}-v^{2}\right)\left(t^{2}-v^{2}\right)}}=\frac{2}{s t} \\
4 \int_{-s}^{s} \frac{d v v^{2}}{\sqrt{\left(s^{2}-v^{2}\right)\left(t^{2}-v^{2}\right)}}=2-2 \alpha \\
\gamma=\frac{\lambda}{16 \pi^{2} L}\left(\frac{1}{s^{2}}+\frac{1}{t^{2}}-2 \frac{1-\alpha}{s t}\right) .
\end{gathered}
$$

In the $\mathrm{SO}(6)$ integrable chain there can be as many as $L$ of the $q$ type roots and $L / 2$ of the $r$ type. In this case, the representation is a singlet. Hence we see that the 
maximum filling fraction is $\alpha=1$, which is reflected in the r.h.s. of (5.5). We also see that $s=1 /(2 \pi)$ when $\alpha=1$. Therefore, the anomalous dimension is

$$
\gamma=\frac{1}{4 L}
$$

which matches the semiclassical prediction for a circular string pulsating on $S_{5}$ [42].

\section{Conclusions}

In this paper we have constructed two types of gauge invariant operators which are in $[J, L-2 J, J]$ representations. The first type is a limit of BMN operators and was shown to have the smallest anomalous dimension for this representation. The second type of solution had a higher anomalous dimension, but was shown to have the same anomalous dimension and fluctuation spectrum as the semiclassical string, strongly suggesting that this is the gauge dual.

The fact that the semiclassical string result in 44 does not correspond to the operator with smallest anomalous dimension is mildly surprising; one usually expects a state with a large amount of symmetry to have a low, if not the lowest energy. It suggests that there are other semiclassical solutions to be found. It also suggests that these semiclassical solutions will involve elliptic integrals in order to reproduce the anomalous dimension.

It would be very important, in order to check and complete our ideas, to find the solution of the Bethe integral equation for the ground state in the case of odd $J$, c.f. section 3.3. Likewise, it would be exciting to find the analog of the purely imaginary solutions for even $J$. On physical grounds, we expect no difference in the energies between even and odd for large $J$, but as we have seen, the root distributions are certainly very different.

The computations presented here are one-loop calculations, but nevertheless not ones that would be accessible to standard Feynman diagram and combinatorial techniques, as the real difficulty is the diagonalization of the states. This nicely illustrates the power of using the Bethe ansatz to do one-loop computations of anomalous dimensions in SYM and suggests that it might be applicable to even more gauge invariant operators. It also illustrates the extremely rich mathematical structure hidden in $\mathcal{N}=4$.

Finally, let us stress the fact that the problems solved in this paper are quite different from the ones considered by condensed matter theorists. For magnetic chains one is either interested in the ferromagnetic or the antiferromagnetic phase. The Bethe vacuum (corresponding to the BPS states in gauge theory) is the true vacuum of the ferromagnet, but only a "fake" vacuum of the antiferromagnet. For finite filling fraction $\alpha$, the twocontour states we considered are hybrids - they are "antiferromagnetic" in the sense that they have low spin, but ferromagnetic in the sense that they have the smallest energy, i.e. anomalous dimension, for fixed $\alpha$. For this reason and to the best of our knowledge, despite all the work on the XXX Heisenberg chain since its invention more than 70 years ago, this problem has not previously been solved.

Note Added: After this paper was completed we learned that Frolov and Tseytlin have studied fluctuations around the stable counterparts of the string soliton dual to the Bethe states discussed here. They found that the $\mathcal{O}(\lambda / L)$ term in the anomalous dimension is indeed not renormalized by the string corrections [55]. 
Second Note Added: After this paper was submitted to the arXiv we were informed that Frolov and Tseytlin were able to find classical string configurations which are dual to the double contour solution presented here. [56].

\section{Acknowledgments}

We are grateful to S. Frolov and A. Tseytlin for correspondence. N.B. and M.S. would like to thank the Department of Theoretical Physics in Uppsala for hospitality. J.A.M. would like to thank the CTP at MIT for hospitality. The work of J.A.M. and K.Z. was supported in part by the Swedish Research Council. The work of K.Z. was also supported in part by RFBR grant 01-01-00549 and in part by grant 00-15-96557 for the promotion of scientific schools. N.B. dankt der Studienstiftung des deutschen Volkes für die Unterstützung durch ein Promotionsförderungsstipendium.

\section{References}

[1] J. M. Maldacena, "The large N limit of superconformal field theories and supergravity", Adv. Theor. Math. Phys. 2 (1998) 231, hep-th/9711200

[2] S. S. Gubser, I. R. Klebanov and A. M. Polyakov, "Gauge theory correlators from non-critical string theory", Phys. Lett. B428 (1998) 105, hep-th/9802109

[3] E. Witten, "Anti-de Sitter space and holography", Adv. Theor. Math. Phys. 2 (1998) 253, hep-th/9802150

[4] A. M. Polyakov, "Gauge fields and space-time", Int. J. Mod. Phys. A17S1 (2002) 119, hep-th/0110196.

[5] D. Berenstein, J. M. Maldacena and H. Nastase, "Strings in flat space and pp waves from $\mathcal{N}=4$ Super Yang Mills", JHEP 0204 (2002) 013, hep-th/0202021.

[6] C. Kristjansen, J. Plefka, G. W. Semenoff and M. Staudacher, "A new double-scaling limit of $\mathcal{N}=4$ super Yang-Mills theory and PP-wave strings", Nucl. Phys. B643 (2002) 3, hep-th/0205033.

[7] D. J. Gross, A. Mikhailov and R. Roiban, "Operators with large $R$ charge in $\mathcal{N}=4$ Yang-Mills theory", Annals Phys. 301 (2002) 31, hep-th/0205066.

[8] N. R. Constable, D. Z. Freedman, M. Headrick, S. Minwalla, L. Motl, A. Postnikov and W. Skiba, "PP-wave string interactions from perturbative Yang-Mills theory", JHEP 0207 (2002) 017, hep-th/0205089.

[9] A. Santambrogio and D. Zanon, "Exact anomalous dimensions of $\mathcal{N}=4$ Yang-Mills operators with large $R$ charge", Phys. Lett. B545 (2002) 425, hep-th/0206079

[10] A. Parnachev and A. V. Ryzhov, "Strings in the near plane wave background and AdS/CFT", JHEP 0210 (2002) 066, hep-th/0208010

[11] U. Gürsoy, "Vector operators in the BMN correspondence", hep-th/0208041.

[12] N. Beisert, C. Kristjansen, J. Plefka, G. W. Semenoff and M. Staudacher, "BMN correlators and operator mixing in $\mathcal{N}=4$ super Yang-Mills theory", Nucl. Phys. B650 (2003) 125, hep-th/0208178.

[13] D. J. Gross, A. Mikhailov and R. Roiban, "A calculation of the plane wave string Hamiltonian from $\mathcal{N}=4$ super-Yang-Mills theory", hep-th/0208231. 
[14] N. R. Constable, D. Z. Freedman, M. Headrick and S. Minwalla, "Operator mixing and the BMN correspondence", JHEP 0210 (2002) 068, hep-th/0209002.

[15] B. Eynard and C. Kristjansen, "BMN correlators by loop equations", JHEP 0210 (2002) 027, hep-th/0209244.

[16] N. Beisert, "BMN Operators and Superconformal Symmetry", Nucl. Phys. B659 (2003) 79, hep-th/0211032.

[17] U. Gürsoy, "Predictions for pp-wave string amplitudes from perturbative SYM", hep-th/0212118.

[18] J. A. Minahan and K. Zarembo, "The Bethe-ansatz for $\mathcal{N}=4$ super Yang-Mills", JHEP 0303 (2003) 013, hep-th/0212208.

[19] N. Beisert, C. Kristjansen, J. Plefka and M. Staudacher, "BMN gauge theory as a quantum mechanical system", Phys. Lett. B558 (2003) 229, hep-th/0212269.

[20] T. Klose, "Conformal Dimensions of Two-Derivative BMN Operators", JHEP 0303 (2003) 012, hep-th/0301150.

[21] N. Beisert, C. Kristjansen and M. Staudacher, "The dilatation operator of $\mathcal{N}=4$ conformal super Yang-Mills theory", Nucl. Phys. B664 (2003) 131, hep-th/0303060.

[22] D. Z. Freedman and U. Gursoy, "Instability and degeneracy in the BMN correspondence", hep-th/0305016.

[23] R. R. Metsaev, "Type IIB Green-Schwarz superstring in plane wave Ramond-Ramond background", Nucl. Phys. B625 (2002) 70, hep-th/0112044.

[24] R. R. Metsaev and A. A. Tseytlin, "Exactly solvable model of superstring in plane wave Ramond-Ramond background", Phys. Rev. D65 (2002) 126004, hep-th/0202109.

[25] M. Spradlin and A. Volovich, "Superstring interactions in a pp-wave background", Phys. Rev. D66 (2002) 086004, hep-th/0204146.

[26] H. Verlinde, "Bits, matrices and 1/N", hep-th/0206059.

[27] M. Spradlin and A. Volovich, "Superstring interactions in a pp-wave background. II", JHEP 0301 (2003) 036, hep-th/0206073.

[28] A. Pankiewicz, "More comments on superstring interactions in the pp-wave background", JHEP 0209 (2002) 056, hep-th/0208209.

[29] J. G. Zhou, "pp-wave string interactions from string bit model", Phys. Rev. D 67, 026010 (2003), hep-th/0208232]

[30] D. Vaman and H. Verlinde, "Bit strings from $\mathcal{N}=4$ gauge theory", hep-th/0209215.

[31] J. Pearson, M. Spradlin, D. Vaman, H. Verlinde and A. Volovich, "Tracing the String: $B M N$ correspondence at Finite $J^{2} / N "$, hep-th/0210102

[32] J. Gomis, S. Moriyama and J. Park, "SYM description of SFT Hamiltonian in a pp-wave background", hep-th/0210153

[33] A. Pankiewicz and B. Stefański, Jr., "PP-Wave Light-Cone Superstring Field Theory", Nucl. Phys. B657 (2003) 79, hep-th/0210246.

[34] R. Roiban, M. Spradlin and A. Volovich, "On light-cone SFT contact terms in a plane wave", hep-th/0211220.

[35] J. Gomis, S. Moriyama and J. Park, "SYM description of pp-wave string interactions: Singlet sector and arbitrary impurities", hep-th/0301250 
[36] M. Spradlin and A. Volovich, "Note on plane wave quantum mechanics", hep-th/0303220.

[37] M. Blau, J. Figueroa-O'Farrill, C. Hull and G. Papadopoulos, "A new maximally supersymmetric background of IIB superstring theory", JHEP 0201 (2002) 047, hep-th/0110242.

[38] M. Blau, J. Figueroa-O'Farrill, C. Hull and G. Papadopoulos, "Penrose limits and maximal supersymmetry", Class. Quant. Grav. 19 (2002) L87, hep-th/0201081

[39] S. S. Gubser, I. R. Klebanov and A. M. Polyakov, "A semi-classical limit of the gauge/string correspondence", Nucl. Phys. B636 (2002) 99, hep-th/0204051

[40] S. Frolov and A. A. Tseytlin, "Semiclassical quantization of rotating superstring in $A d S_{5} \times S^{5}$ ", JHEP 0206 (2002) 007, hep-th/0204226.

[41] J. G. Russo, "Anomalous dimensions in gauge theories from rotating strings in $A d S_{5} \times S^{5}$ ", JHEP 0206 (2002) 038, hep-th/0205244.

[42] J. A. Minahan, "Circular semiclassical string solutions on $A d S_{5} \times S^{5}$ ", Nucl. Phys. B648 (2003) 203, hep-th/0209047.

[43] A. A. Tseytlin, "Semiclassical quantization of superstrings: $A d S_{5} \times S^{5}$ and beyond", Int. J. Mod. Phys. A18 (2003) 981, hep-th/0209116.

[44] S. Frolov and A. A. Tseytlin, "Multi-spin string solutions in $A d S_{5} \times S^{5}$ ", hep-th/0304255.

[45] M. R. Douglas and V. A. Kazakov, "Large $N$ phase transition in continuum QCD in two-dimensions", Phys. Lett. B319 (1993) 219, hep-th/9305047.

[46] V. A. Kazakov, M. Staudacher and T. Wynter, "Character expansion methods for matrix models of dually weighted graphs", Commun. Math. Phys. 177 (1996) 451, hep-th/9502132.

[47] L. D. Faddeev, "How Algebraic Bethe Ansatz works for integrable model", hep-th/9605187.

[48] I. K. Kostov and M. Staudacher, "Multicritical phases of the $O(n)$ model on a random lattice", Nucl. Phys. B384 (1992) 459, hep-th/9203030.

[49] F. David, "Phases of the large $N$ matrix model and nonperturbative effects in 2-d gravity", Nucl. Phys. B348 (1991) 507.

[50] H. Bethe, "On the theory of metals. 1. Eigenvalues and eigenfunctions for the linear atomic chain", Z. Phys. 71 (1931) 205.

[51] F. H. L. Essler, V. E. Korepin and K. Schoutens, "Fine structure of the Bethe ansatz for the spin 1/2 Heisenberg XXX model", J. Phys. A25 (1992) 4115.

[52] A. N. Kirillov and N. A. Liskova, "Completeness of Bethe's states for generalized XXZ model", J. Phys. A30 (1997) 1209, hep-th/9403107.

[53] G. Juttner and M. Karowski, "Completeness of 'good' Bethe Ansatz solutions of a quantum group invariant Heisenberg Model", Nucl. Phys. B430 (1994) 615, hep-th/9406183.

[54] R. J. Baxter, "Completeness of the Bethe ansatz for the six and eight-vertex models", cond-mat/0111188.

[55] S. Frolov and A. A. Tseytlin, "Quantizing three-spin string solution in $A d S_{5} \times S^{5}$ ", hep-th/0306130. 
[56] S. Frolov and A. A. Tseytlin, "Rotating string solutions: AdS/CFT duality in non-supersymmetric sectors", hep-th/0306143. 


\section{Tables}

\begin{tabular}{|c|c|c|c|}
\hline$\overline{\gamma\left[\lambda J / L^{2}\right]}$ & \multicolumn{2}{|l|}{ Bethe roots } & $n$ \\
\hline 0.675927 & $( \pm 0.676245 \pm 0.993633 i)$ & \pm 0.678017 & 111 \\
\hline 0.948774 & $( \pm 0.120258 \pm 0.500000 i)$ & $\pm 1.638344 i$ & 220 \\
\hline 1.485952 & $\pm 2.177728 i \quad \pm 1.000078 i$ & \pm 0 . & 005 \\
\hline 1.913430 & $( \pm 0.347918 \pm 0.500019 i)$ & \pm 0.695172 & 221 \\
\hline 2.303250 & $\pm 1.003317 i \quad \pm 0.805340$ & \pm 0.012646 & 015 \\
\hline 2.804933 & $( \pm 0.519954 \pm 0.500990 i)$ & \pm 0.2 & 114 \\
\hline 3.163442 & $\pm 1.012980 i \quad \pm 0.386936$ & \pm 0.024593 & 025 \\
\hline 3.283924 & $( \pm 0.560191 \pm 0.501721 i)$ & \pm 0.083335 & 115 \\
\hline 3.854384 & $\pm 1.063338 i \quad \pm 0.186496$ & \pm 0.047508 & 035 \\
\hline 5.098922 & \pm 0.657299 & \pm 0.084694 & 135 \\
\hline
\end{tabular}

Table 5: Unpaired states of $[6,0,6]$

\begin{tabular}{|c|cccc|c|}
\hline$\gamma\left[\lambda J / L^{2}\right]$ & $|c|$ & $n$ \\
\hline 0.815506 & 0 & $\pm i / 2$ & $\pm 1.000016 i$ & $\pm 1.478037 i$ & $6 * 11$ \\
1.033467 & 0 & $\pm i / 2$ & $\pm 0.999996 i$ & $\pm 2.241154 i$ & $5 * 10$ \\
1.684344 & 0 & $\pm i / 2$ & $\pm 1.500899 i$ & $\pm 2.862938 i$ & $6 * 00$ \\
1.870623 & 0 & $\pm i / 2$ & $\pm 0.999885 i$ & \pm 0.882971 & $5 * 11$ \\
2.366246 & 0 & $\pm i / 2$ & $\pm 1.524153 i$ & \pm 0.985655 & $6 * 01$ \\
2.749876 & 0 & $\pm i / 2$ & $\pm 0.999612 i$ & \pm 0.449560 & $5 * 12$ \\
3.065353 & 0 & $\pm i / 2$ & $( \pm 0.701060 \pm 0.504190 i)$ & $6 * 11$ \\
3.156982 & 0 & $\pm i / 2$ & $\pm 1.555226 i$ & \pm 0.522436 & $6 * 02$ \\
3.514967 & 0 & $\pm i / 2$ & $\pm 0.998615 i$ & \pm 0.231443 & $5 * 13$ \\
3.915465 & 0 & $\pm i / 2$ & $\pm 1.584609 i$ & \pm 0.295822 & $6 * 03$ \\
3.939509 & 0 & $\pm i / 2$ & $\pm 0.986006 i$ & \pm 0.062156 & $5 * 14$ \\
4.462322 & 0 & $\pm i / 2$ & $\pm 1.603217 i$ & \pm 0.136490 & $6 * 04$ \\
4.926542 & 0 & $\pm i / 2$ & \pm 0.740432 & \pm 0.348252 & $6 * 13$ \\
5.587007 & 0 & $\pm i / 2$ & \pm 0.752202 & \pm 0.154446 & $6 * 14$ \\
6.559173 & 0 & $\pm i / 2$ & \pm 0.364262 & \pm 0.158450 & $6 * 24$ \\
\hline
\end{tabular}

Table 6: Unpaired states of $[7,0,7]$ 